Research article

\title{
Evidence for association between the HLA-DQA locus and abdominal aortic aneurysms in the Belgian population: a case control study
}

\author{
Toru Ogata ${ }^{1}$, Lucie Gregoire ${ }^{2}$, Katrina AB Goddard ${ }^{3}$, Magdalena Skunca ${ }^{1}$, \\ Gerard Tromp ${ }^{1}$, Wayne D Lancaster ${ }^{1,4}$, Antonio R Parrado ${ }^{3}$, Qing Lu ${ }^{3}$, \\ Hidenori Shibamura1 ${ }^{1}$ Natzi Sakalihasan ${ }^{5}$, Raymond Limet ${ }^{5}$, \\ Gerald L MacKean ${ }^{6}$, Claudette Arthur ${ }^{6}$, Taijiro Sueda ${ }^{7}$ and \\ Helena Kuivaniemi*1,8
}

\begin{abstract}
Address: ${ }^{1}$ Center for Molecular Medicine and Genetics, Wayne State University School of Medicine, Detroit, Michigan, USA, ${ }^{2}$ Department of Immunology and Microbiology, Wayne State University School of Medicine, Detroit, Michigan, USA, ${ }^{3}$ Department of Epidemiology and Biostatistics, Case Western Reserve University, Cleveland, Ohio, USA, ${ }^{4}$ Department of Obstetrics and Gynecology, Wayne State University School of Medicine, Detroit, Michigan, USA, ${ }^{5}$ Department of Cardiovascular Surgery, University of Liège, Liège, Belgium, ${ }^{6}$ Department of Surgery, Dalhousie University, Halifax, Canada, ${ }^{7}$ Department of Surgery, Graduate School of Biomedical Science, Hiroshima University, Hiroshima, Japan and ${ }^{8}$ Department of Surgery, Wayne State University School of Medicine, Detroit, Michigan, USA

Email: Toru Ogata - ogatat@hiroshima-u.ac.jp; Lucie Gregoire - gregoire@genetics.wayne.edu; Katrina AB Goddard - katrina@darwin.epbi.cwru.edu; Magdalena Skunca - ac3610@wayne.edu; Gerard Tromp - tromp@sanger.med.wayne.edu; Wayne D Lancaster - wayne@genetics.wayne.edu;

Antonio R Parrado - tparrado@darwin.cwru.edu; Qing Lu - qlu@darwin.cwru.edu; Hidenori Shibamura - h-shiba@asa-hosp.city.hiroshima.jp; Natzi Sakalihasan - nsaka@chu.ulg.ac.be; Raymond Limet - rlimet@chu.ulg.ac.be; Gerald L MacKean - g.mackean@dal.ca;

Claudette Arthur - Claudette.Arthur@dal.ca; Taijiro Sueda - sueda@ hiroshima-u.ac.jp; Helena Kuivaniemi* - kuivan@sanger.med.wayne.edu

* Corresponding author
\end{abstract}

Published: 31 July 2006

BMC Medical Genetics 2006, 7:67 doi:10.1 186/147|-2350-7-67
Received: 18 May 2006

Accepted: 31 July 2006

This article is available from: http://www.biomedcentral.com/I47/-2350/7/67

(C) 2006 Ogata et al; licensee BioMed Central Ltd.

This is an Open Access article distributed under the terms of the Creative Commons Attribution License (http://creativecommons.org/licenses/by/2.0), which permits unrestricted use, distribution, and reproduction in any medium, provided the original work is properly cited.

\footnotetext{
Abstract

Background: Chronic inflammation and autoimmunity likely contribute to the pathogenesis of abdominal aortic aneurysms (AAAs). The aim of this study was to investigate the role of autoimmunity in the etiology of AAAs using a genetic association study approach with HLA polymorphisms.

Methods: HLA-DQAI, -DQBI, -DRBI and -DRB3-5 alleles were determined in 387 AAA cases ( 180 Belgian and 207 Canadian) and 426 controls (269 Belgian and I57 Canadian) by a PCR and single-strand oligonucleotide probe hybridization assay.

Results: We observed a potential association with the HLA-DQAI locus among Belgian males (empirical $p=0.027$, asymptotic $p=0.071)$. Specifically, there was a significant difference in the HLA-DQAI $* 0102$ allele frequencies between AAA cases (67/322 alleles, 20.8\%) and controls (44/356 alleles, 12.4\%) in Belgian males (empirical $p=0.019$, asymptotic $p=0.003$ ). In haplotype analyses, marginally significant association was found between AAA and haplotype HLA-DQAI-DRBI $(p=0.049$ with global score statistics and $p=$ 0.002 with haplotype-specific score statistics).

Conclusion: This study showed potential evidence that the HLA-DQAI locus harbors a genetic risk factor for AAAs suggesting that autoimmunity plays a role in the pathogenesis of AAAs.
} 


\section{Background}

Several distinct processes contribute to the pathologic changes observed in abdominal aortic aneurysms (AAAs). The most apparent of these are chronic inflammation, destructive remodeling of the extracellular matrix, and depletion of vascular smooth muscle cells [1]. Local immune responses in the aorta are an important factor in AAA pathogenesis. Autoimmunity has been proposed to play a role in the pathogenesis of AAA [2,3]. Infiltration of monocytes, macrophages, B-lymphocytes, plasma cells and T-lymphocytes (including both CD4 and CD8 T-cells) is commonly observed in the AAA walls [4]. Although the actual factors (triggers) responsible for initiating the chronic inflammatory response in the pathogenesis of AAA are not yet known, HLA loci, particularly the HLADQ and HLA-DR antigens, may play a key role.

The major histocompatibility complex (MHC) is located at chromosome 6p21.31 and is the most gene-dense and polymorphic region of the human genome identified so far [5]. Historically, the MHC has been divided into three regions: HLA class I, class II and class III. Although class I antigens are present on the surface of most types of cells in the human body, class II antigens are expressed by a few types of antigen-presenting cells, namely B-lymphocytes, macrophages, dendritic cells, thymic epithelial cells, and activated T lymphocytes [6,7]. The MHC locus has been associated with more diseases than any other region of the human genome with more than 20,000 research articles published [8], and most of the significant associations were with the class II polymorphisms $[5,7]$.

The HLA class II region contains five isotypes, HLA-DM, DO, -DP, -DQ and -DR, all of which are heterodimers composed of $\alpha$ and $\beta$ chains [7]. There are only few polymorphisms in the HLA-DM and -DO isoforms. On the other hand, HLA-DP, -DQ and -DR are quite polymorphic [7]. For HLA-DQ, both the $\alpha$ and $\beta$ chains, which are expressed by HLA-DQA1 and -DQB1 genes, respectively, contribute to the variability. For HLA-DRB, only the $\beta$ chain, which is expressed by the HLA-DRB1 gene, contributes to the variability [7]. HLA-DRB1 ( $\beta$ chain) has another functional isoform, DRB3-5, whose genes are located close to the -DRB1 gene. Only one allele in each individual is expressed from DRB3, 4 and 5 genes combined [7]. HLA-DQ and -DR proteins are responsible for presenting foreign peptide antigens from infectious agents, such as bacteria, viruses or autoimmune antigens, to CD4 T-cells. These antigens stimulate CD4 T-cell responses that activate $\mathrm{B}$-cells and macrophages. The structure of the HLA-DQ or -DR peptide-binding groove varies considerably depending on which DQA1, DQB1 and DRB1 alleles are being exposed [9]. These genetic differences may affect the immune response by increasing or decreasing the ability of HLA-DQ or -DR molecules to bind and properly present foreign antigens to the CD4 Tcell $[7,10]$.

Several association studies between HLA polymorphisms and AAAs have been performed [11-19]. The sample sizes of these studies, however, were small and the results were inconsistent. The aim of the current study was to investigate further the role of autoimmunity in the etiology of AAAs by carrying out a genetic association study with the HLA-DQA1, -DQB1, and -DRB polymorphisms for AAA.

\section{Methods \\ Study population}

A definition of AAA by Johnston et al [20] (a diameter of infrarenal aorta $\geq 3 \mathrm{~cm}$ ) was used. These standards have also been used by other investigators [21-23]. Altogether 387 unrelated AAA cases (males: 316, 81.7\%), 180 Belgian (males: 161, 89.4\%), admitted to University Hospital of Liège in Liège, and 207 Canadian (males: 155, 74.9\%), admitted to Dalhousie University Hospital in Halifax, were entered into the study. Seventeen patients were admitted for emergency repair of ruptured AAA and 335 patients were admitted for elective surgery. Thirty-five patients were diagnosed with AAA using ultrasonography and were not operated on due to old age or because the size of the aneurysm was relatively small $(<5 \mathrm{~cm})$. Altogether 152 cases (39.3\%) had a family history of AAA, which was defined as having at least one first-degree relative affected with AAA. All patients were Caucasian.

Control samples were obtained from 426 Caucasians (males: $\mathrm{n}=217,50.9 \%$; 269 Belgian and 157 Canadian) and included spouses of AAA cases $(\mathrm{n}=114$; all Canadian; 4 males, 110 females), or individuals admitted to the same hospitals for reasons other than AAA ( $\mathrm{n}=312 ; 183$ Belgian males, 86 Belgian females; 29 Canadian males and 14 Canadian females).

The study was approved by the Institutional Review Boards of Wayne State University School of Medicine and each patient recruiting center. All subjects gave informed written consent to participate into the study.

\section{Genotyping}

Genomic DNA was isolated from peripheral blood using a Puregene kit (Gentra Systems, Minneapolis, MN). Before performing genotyping PCR, a whole-genome amplification using primer extension preamplification (PEP) was carried out to increase the amount of template DNA available for genotyping and to ensure that limited resources are used cost-effectively [24]. The performance of PEP in subsequent genotyping reactions was validated extensively in our laboratory previously [24]. The PEP products were diluted 100-fold and used for genotyping. Throughout the manuscript we use the gene symbols 
approved by the HUGO Gene Nomenclature Committee [25].

HLA-DQA1, -DQB1, -DRB1 and -DRB3-5 alleles were determined by PCR and single-strand oligonucleotide probe hybridization assay. HLA-DQA1 alleles were determined according to Kimura et al [26]. Quality control samples, including positive and negative controls, were included in each set of 96 samples run in genotyping reactions. Positive controls included samples whose HLA genotypes were known. HLA-DQA1 was amplified using primers DQAP1 (5'-ATGGTGTAAACTTGTACCAGT-3') and DQAP2 (5'-TTGGTAGCAGCGGTAGAGTTG-3') to generate a 230-bp fragment. Fourteen single-strand oligonucleotide probes discriminated between HLADQA $1{ }^{*} 0101 / 0104,{ }^{*} 0102,{ }^{*} 0103,{ }^{*} 0201,{ }^{*} 03011 / 0302$, ${ }^{*} 03012,{ }^{*} 0401,{ }^{*} 0501$ and ${ }^{*} 0601$ alleles. HLA-DQB1 alleles were determined as previously described [27]. HLA-DQB1 was amplified using primers DQP14 (5'-TGTGCTACTTCACCAACGGG-3') and DQP5 (5'-GGTAGTTGTGTCTGCACAC-3') to generate a 210-bp fragment. Twenty-five single-strand oligonucleotide probes could discriminate between HLA-DQB1*0201/0202, *0301, *0302, *03031, *03032 *0304, *0305, *0401, *0402, ${ }^{*} 0501,{ }^{*} 0502,{ }^{*} 0503,{ }^{*} 0504,{ }^{*} 0601,{ }^{*} 0602,{ }^{*} 0603$, ${ }^{*} 0604, * 0605, * 0606, * 0607$ and ${ }^{*} 0608$ alleles. Both HLA-DRB1 and DRB3-5 alleles were amplified using primers GH46 (5'-CCGGATCCTTCGTGTCCCCACAGCACG-3') and P2A (5'-TCGCCGCTGCACTGTGAAGC-3') to generate a 290-bp fragment. Forty single-strand oligonucleotide probes could discriminate between HLADRB1*0101, *0102, *0103, *0301/0303, *0302, *0401, *0402, *0403, *0404, *0405, *0406, *0407, *0408, *0409, *0410, *0411, *0412, *0413, *0414, *0415, *0701/0702, *0801, *0802, *0803, *0804, *0805, ${ }^{*} 0806,{ }^{*} 0901,{ }^{*} 1001,{ }^{*} 1101,{ }^{*} 1102,{ }^{*} 1103,{ }^{*} 1104$, ${ }^{*} 1105, * 1201, * 1202,{ }^{*} 1301,{ }^{*} 1302,{ }^{*} 1303, * 1304$, ${ }^{*} 1305, * 1306, * 1401,{ }^{*} 1402,{ }^{*} 1403,{ }^{*} 1404, * 1405$, *1406, *1407, *1408, *1409, *1410, *1501, *1502, ${ }^{*} 1503,{ }^{*} 1601$ and ${ }^{*} 1602$ as well as DRB3*0101, ${ }^{*} 0201$, *0202, *0301, DRB4*0101, DRB5*0101, *0102, *0201/ 0202 and ${ }^{*} 0203$ alleles [26]. Samples whose hybridization pattern failed to match with the pattern of a known allele were classified as "could not be determined". Exceptions were the unknown alleles which could be classified into two possible alleles, such as HLA-DRB ${ }^{*} 0403$ or *0407, but could not be classified into one particular allele, and were then designated as $" * 0403$ or ${ }^{*} 0407 "$. Two individuals (T.O. and L.G.) scored the results independently and discrepancies were resolved by re-evaluation of the raw data or repeating the experiment, if necessary.

\section{Statistical analyses}

First, the distributions of allele frequencies between the Belgian and Canadian control groups were compared. Asymptotic p-values were obtained using the $\chi^{2}$ test for association. Because of the large number of alleles at each locus, we also obtained empirical p-values for the test of association for each allele using a permutation test for the entire table (called the T1 test statistic as opposed to other test statistics computed by this program that collapses the table in various ways) as implemented in the computer program CLUMP [28]. In this permutation test, the population group is permuted numerous times, and the test statistic is recomputed for each permutation. The observed test statistic is then compared to the distribution of the permuted test statistics to obtain an empirical pvalue. Significant differences between the two control groups for all of the HLA loci were found. The p-values were: 0.062 (empirical: 0.056) for DQA; 0 (empirical: 0.0001) for DQB, 0.008 (empirical: 0.0046) for DRB, and 0 (empirical: 0.0001) for DRB3-5. Canadian and Belgian populations were, therefore, analyzed separately. To evaluate the association between alleles and AAA, the same approach was used. The Haplo.stats program [29] was used to estimate haplotype frequencies via the expectation-maximization (EM) algorithm for HLA-DQA1, DQB1, -DRB1 and -DRB3-5 alleles, and to compute two score tests of association between disease status and HLA haplotype: 1) global score statistics and 2) haplotype-specific score statistics. Rare haplotypes with frequencies $\leq$ 0.01 were pooled into a single baseline group. The score test is developed under the generalized linear model framework, where the score function is the first derivative of the log-likelihood from the exponential family distribution. The variance matrix is determined by the matrix of negative second partial derivative of the log-likelihood. Using the results from the EM algorithm, the score statistics $S=U^{T} V^{-1} U$ are evaluated under the null hypothesis, which asymptotically has a chi-square distribution. Empirical p-values are obtained for the score statistics using permutation. Results on haplotypes with only two loci are reported, since the contingency table for 3- or 4locus results was sparse given the large number of alleles at each locus. Analyses were also conducted stratified by sex, and the presence of a positive family history. Pairwise measures of linkage disequilibrium (LD) were estimated using the observed genotypes for the four HLA loci using the GOLD program [30]. All measures of $\mathrm{D}^{\prime}$ were between 0.26 and 0.37 , indicating comparatively low LD between loci. Correction for multiple testing was applied to the most significant p-values using the Sidak function.

\section{Post hoc power calculations}

Since the HLA locus has a large number of alleles and the allele frequencies are variable across populations, we computed power for the locus based on the frequency 
observed in the samples. Power calculations were performed using the power calculator for binomial proportions in Splus 7.0 (Insightful Corporation, Seattle, WA) for a test of the null hypothesis that $\mathrm{p} 1=\mathrm{p} 2$, where $\mathrm{p} 1$ is the allele frequency in cases and $\mathrm{p} 2$ is the allele frequency in controls, versus the alternative hypothesis that the allele frequencies for the cases and controls are different. To obtain $80 \%$ power with the risk allele frequency of 0.208 in the cases and 0.124 in the controls, we needed 331 subjects per group for an $\alpha$ of 0.05 , and 460 subjects per group for an $\alpha$ of 0.0125 (to account for multiple testing).

\section{Results}

HLA-DQA1, -DQB1, -DRB1 and -DRB3-5 allele frequencies are summarized in Tables 1, 2, 3, 4. Empirical p-values obtained by permutation test and asymptotic p-values obtained by $\chi^{2}$ test are shown in Table 5. Results are shown separately for the Belgian and Canadian populations for two reasons: 1) comparison of the distribution of allele frequencies between the Belgian and Canadian control groups using a chi-square test showed significant differences for all of the HLA loci. The p-values were: 0.062 (empirical: 0.056) for DQA; 0 (empirical: 0.0001) for DQB, 0.008 (empirical: 0.0046) for DRB, and 0 (empirical: 0.0001) for DRB3-5; and 2) in our previous work we showed highly significant departure from Hardy-Weinberg proportions for several markers in the combined sample, but not in the subsets, indicating evidence for the presence of population stratification [31]. There was a nominally significant association between AAA and HLADQA1 locus in the Belgian population (empirical $\mathrm{p}=$ 0.039 ; asymptotic $\mathrm{p}=0.049 ; \mathrm{p}=0.147$ after Sidak correction for multiple testing). In particular, the frequency of the HLA-DQA $1 * 0102$ allele was significantly increased in the AAA group (75/360 alleles, 20.8\%) compared to the

Table I: HLA-DQAI allele frequencies in AAA cases and controls

\begin{tabular}{|c|c|c|c|c|c|c|c|c|c|c|c|c|}
\hline \multirow[b]{3}{*}{$\begin{array}{l}\text { DQAI } \\
\text { alleles }\end{array}$} & \multicolumn{6}{|c|}{ AAA Cases } & \multicolumn{6}{|c|}{ Controls } \\
\hline & \multicolumn{2}{|c|}{ Male } & \multicolumn{2}{|c|}{ Female } & \multicolumn{2}{|c|}{ Total } & \multicolumn{2}{|c|}{ Male } & \multicolumn{2}{|c|}{ Female } & \multicolumn{2}{|c|}{ Total } \\
\hline & $\mathrm{n}^{\mathrm{a}}$ & $\%$ & $\mathrm{n}^{\mathrm{a}}$ & $\%$ & $\mathrm{n}^{\mathrm{a}}$ & $\%$ & $\mathrm{n}^{\mathrm{a}}$ & $\%$ & $\mathrm{n}^{\mathrm{a}}$ & $\%$ & $\mathrm{n}^{\mathrm{a}}$ & $\%$ \\
\hline \multicolumn{13}{|l|}{ Belgian } \\
\hline $\begin{array}{l}* 0101 / \\
0104\end{array}$ & 45 & 14.0 & 4 & 10.5 & 49 & 13.6 & 68 & 19.1 & 22 & 13.3 & 90 & 17.2 \\
\hline *0102 & 67 & $20.8^{b}$ & 8 & 21.1 & 75 & $20.8^{c}$ & 44 & $12.4^{b}$ & 27 & 16.3 & 71 & $13.6^{c}$ \\
\hline *0103 & 25 & 7.8 & I & 2.6 & 26 & 7.2 & 36 & 10.1 & 8 & 4.8 & 44 & 8.4 \\
\hline *020I & 53 & 16.5 & 3 & 7.9 & 56 & 15.6 & 46 & 12.9 & 19 & 11.4 & 65 & 12.5 \\
\hline $\begin{array}{l}* 03011 / \\
0302\end{array}$ & 37 & 11.5 & 5 & 13.2 & 42 & 11.7 & 54 & 15.2 & 30 & 18.1 & 84 & 16.1 \\
\hline$* 03012$ & 0 & 0 & 0 & 0 & 0 & 0 & 0 & 0 & 1 & 0.6 & 1 & 0.2 \\
\hline *0401 & 8 & 2.5 & 3 & 7.9 & 11 & 3.1 & 11 & 3.1 & 8 & 4.8 & 19 & 3.6 \\
\hline$* 0501$ & 87 & 27.0 & 14 & 36.8 & 101 & 28.1 & 97 & 27.2 & 49 & 29.5 & 146 & 28.0 \\
\hline$* 0601$ & 0 & 0 & 0 & 0 & 0 & 0 & 0 & 0 & 2 & 1.2 & 2 & 0.4 \\
\hline $\begin{array}{l}\text { Total } \\
\text { Canadian }\end{array}$ & 322 & 100.0 & 38 & 100.0 & 360 & 100.0 & 356 & 100.0 & 166 & 100.0 & 522 & 100.0 \\
\hline $\begin{array}{l}* 0101 / \\
0104\end{array}$ & 46 & 14.9 & 7 & 7.3 & 53 & 13.1 & 12 & 18.2 & 42 & 17.1 & 54 & 17.3 \\
\hline *0102 & 58 & 18.8 & 20 & 20.8 & 78 & 19.3 & 11 & 16.7 & 37 & 15.0 & 48 & 15.4 \\
\hline$* 0103$ & 11 & 3.6 & 7 & 7.3 & 18 & 4.6 & 4 & 6.1 & 15 & 6.1 & 19 & 6.1 \\
\hline *020I & 48 & 15.6 & 17 & 17.7 & 65 & 15.8 & 17 & 25.8 & 34 & 13.8 & 51 & 16.3 \\
\hline $\begin{array}{l}* 03011 / 1 \\
0302\end{array}$ & 66 & 21.4 & 19 & 19.8 & 85 & 21.0 & 12 & 18.2 & 51 & 20.7 & 63 & 20.2 \\
\hline$* 03012$ & 0 & 0 & 0 & 0 & 0 & 0 & 0 & 0 & 0 & 0 & 0 & 0 \\
\hline *040I & 7 & 2.3 & 2 & 2.1 & 9 & 2.2 & 1 & 1.5 & 9 & 3.7 & 10 & 3.2 \\
\hline$* 0501$ & 71 & 23.1 & 24 & 25.0 & 95 & 23.5 & 9 & 13.6 & 57 & 23.2 & 66 & 21.2 \\
\hline *060I & 1 & 0.3 & 0 & 0 & 1 & 0.2 & 0 & 0 & 1 & 0.4 & I & 0.3 \\
\hline Total & 308 & 100.0 & 96 & 100.0 & 404 & 100.0 & 66 & 100.0 & 246 & 100.0 & 312 & 100.0 \\
\hline
\end{tabular}

AAA: abdominal aortic aneurysm.

a Number of alleles.

${ }^{b}$ Empirical $P=0.019$, asymptotic $P=0.003$ for comparison of this particular allele versus all other alleles combined between cases and controls among Belgian males.

c Empirical $P=0.033$, asymptotic $P=0.005$ for comparison of this particular allele versus all other alleles combined between cases and controls among Belgians. 
Table 2: HLA-DQB I allele frequencies in AAA cases and controls

\begin{tabular}{|c|c|c|c|c|c|c|c|c|c|c|c|c|}
\hline \multirow[b]{3}{*}{ DQBI alleles ${ }^{\mathrm{a}}$} & \multicolumn{6}{|c|}{ AAA Cases } & \multicolumn{6}{|c|}{ Controls } \\
\hline & \multicolumn{2}{|c|}{ Male } & \multicolumn{2}{|c|}{ Female } & \multicolumn{2}{|c|}{ Total } & \multicolumn{2}{|c|}{ Male } & \multicolumn{2}{|c|}{ Female } & \multicolumn{2}{|c|}{ Total } \\
\hline & $n^{b}$ & $\%$ & $n^{b}$ & $\%$ & $n^{b}$ & $\%$ & $n^{b}$ & $\%$ & $n^{b}$ & $\%$ & $n^{b}$ & $\%$ \\
\hline \multicolumn{13}{|l|}{ Belgian } \\
\hline *0201/0202 & 62 & 19.4 & 5 & 15.2 & 67 & 19.0 & 72 & 21.4 & 36 & 21.8 & 108 & 21.5 \\
\hline$*_{0301}$ & 59 & 18.5 & 9 & 27.2 & 68 & 19.3 & 68 & 20.2 & 46 & 27.9 & 114 & 22.7 \\
\hline *0302 & 32 & 10.0 & 3 & 9.1 & 35 & 10.0 & 20 & 5.9 & 10 & 6.1 & 30 & 6.0 \\
\hline *03031 & 0 & 0 & 0 & 0 & 0 & 0 & 7 & 2.1 & 2 & 1.2 & 9 & 1.8 \\
\hline *03032 & 10 & 3.1 & 0 & 0 & 10 & 2.8 & 8 & 2.4 & 5 & 3.0 & 13 & 2.6 \\
\hline *0304 & 8 & 2.5 & 3 & 9.1 & 11 & 3.1 & 7 & 2.1 & 4 & 2.4 & 11 & 2.2 \\
\hline *0305 & 3 & 0.9 & 0 & 0 & 3 & 0.9 & 1 & 0.3 & 0 & 0 & 1 & 0.2 \\
\hline$* 0401$ & 10 & 3.1 & 3 & 9.1 & 13 & 3.7 & 10 & 3.0 & 8 & 4.8 & 18 & 3.6 \\
\hline *0501 & 40 & 12.5 & 4 & 12.1 & 44 & 12.5 & 56 & 16.6 & 15 & 9.1 & 71 & 14.1 \\
\hline *0502 & 7 & 2.2 & 0 & 0 & 7 & 2.0 & 5 & 1.5 & 4 & 2.4 & 9 & 1.8 \\
\hline$* 0503$ & 9 & 2.8 & 0 & 0 & 9 & 2.6 & 8 & 2.4 & 7 & 4.2 & 15 & 3.0 \\
\hline$* 0601$ & 0 & 0 & 0 & 0 & 0 & 0 & 1 & 0.3 & 0 & 0 & 1 & 0.2 \\
\hline$* 0602$ & 41 & 12.9 & I & 3.0 & 42 & 12.0 & 24 & 7.1 & 14 & 8.5 & 38 & 7.6 \\
\hline$* 0603$ & 23 & 7.2 & 3 & 9.1 & 26 & 7.4 & 33 & 9.8 & 6 & 3.6 & 39 & 7.8 \\
\hline$* 0604$ & 13 & 4.1 & 2 & 6.1 & 15 & 4.3 & 12 & 3.6 & 5 & 3.0 & 17 & 3.4 \\
\hline *0605/0609 & 1 & 0.3 & 0 & 0 & I & 0.3 & 3 & 0.9 & 2 & 1.2 & 5 & 1.0 \\
\hline$* 0606$ & 0 & 0 & 0 & 0 & 0 & 0 & 0 & 0 & 1 & 0.6 & 1 & 0.2 \\
\hline$* 0607$ & 1 & 0.3 & 0 & 0 & 1 & 0.3 & 2 & 0.6 & 0 & 0 & 2 & 0.4 \\
\hline Total & 319 & 100.0 & 33 & 100.0 & 352 & 100.0 & 337 & 100.0 & 165 & 100.0 & 502 & 100.0 \\
\hline \multicolumn{13}{|l|}{ Canadian } \\
\hline *0201/0202 & 77 & 25.1 & 26 & 25.5 & 103 & 25.2 & 19 & 28.8 & 63 & 25.6 & 82 & 26.3 \\
\hline$* 0301$ & 54 & 17.6 & 20 & 19.6 & 74 & 18.1 & 9 & 13.6 & 42 & 17.1 & 51 & 16.3 \\
\hline *0302 & 35 & 11.4 & 11 & 10.8 & 46 & 11.2 & 8 & 12.1 & 30 & 12.2 & 38 & 12.2 \\
\hline *03031 & 5 & 1.6 & 0 & 0 & 5 & 1.2 & 0 & 0 & 1 & 0.4 & 1 & 0.3 \\
\hline *03032 & 16 & 5.2 & 5 & 4.9 & 21 & 5.1 & 4 & 6.0 & 6 & 2.4 & 10 & 3.2 \\
\hline *0304 & 4 & 1.3 & 0 & 0 & 4 & 1.0 & 0 & 0 & 3 & 1.2 & 3 & 1.0 \\
\hline$* 0305$ & 0 & 0 & 0 & 0 & 0 & 0 & 0 & 0 & 0 & 0 & 0 & 0 \\
\hline$* 0401$ & 6 & 2.0 & I & 1.0 & 7 & 1.7 & I & 1.5 & 8 & 3.3 & 9 & 2.9 \\
\hline$* 0501$ & 38 & 12.4 & 8 & 7.8 & 46 & 11.2 & 8 & 12.1 & 33 & 13.4 & 41 & 13.1 \\
\hline$* 0502$ & 6 & 2.0 & I & 1.0 & 7 & 1.7 & 0 & 0 & 0 & 0 & 0 & 0 \\
\hline$* 0503$ & 8 & 2.6 & i & 1.0 & 9 & 2.2 & 3 & 4.5 & 9 & 3.7 & 12 & 3.8 \\
\hline$* 0601$ & 2 & 0.7 & 1 & 1.0 & 3 & 0.7 & 0 & 0 & 1 & 0.4 & 1 & 0.3 \\
\hline$* 0602$ & 31 & 10.1 & 18 & 17.6 & 49 & 12.0 & 9 & 13.6 & 25 & 10.2 & 34 & 10.9 \\
\hline$* 0603$ & 12 & 3.9 & 6 & 5.9 & 18 & 4.4 & 4 & 6.0 & 14 & 5.7 & 18 & 5.8 \\
\hline *0604 & 10 & 3.3 & 3 & 2.9 & 13 & 3.2 & I & 1.5 & 9 & 3.7 & 10 & 3.2 \\
\hline *0605/0609 & 3 & 1.0 & 1 & 1.0 & 4 & 1.0 & 0 & 0 & 1 & 0.4 & 1 & 0.3 \\
\hline$* 0606$ & 0 & 0 & 0 & 0 & 0 & 0 & 0 & 0 & 0 & 0 & 0 & 0 \\
\hline$* 0607$ & 0 & 0 & 0 & 0 & 0 & 0 & 0 & 0 & 1 & 0.4 & I & 0.3 \\
\hline Total & 307 & 100.0 & 102 & 100.0 & 409 & 100.0 & 66 & 100.0 & 246 & 100.0 & 312 & 100.0 \\
\hline
\end{tabular}

AAA: abdominal aortic aneurysm.

a Alleles $* 0402, * 0504$ and $* 0608$ were also assayed but were not present in either population.

b Number of alleles.

control group (71/522 alleles, $13.6 \%)$ in the Belgian population (empirical $\mathrm{p}=0.033$; asymptotic $\mathrm{p}=0.005$; Table $1)$. No significant associations were found in the Canadian population (Tables 1, 2, 3, 4, 5), but this might be due to lower power to detect such associations because of smaller sample size.

The results on analyses in which the AAA cases and controls were stratified by sex are shown in Table 5. The HLA-
DQA1 allele frequencies in AAA cases and controls in the Belgians stratified by sex are shown in Table 1 . The association between AAA and HLA-DQA1 locus remained nominally significant only in the Belgian male population (empirical $\mathrm{p}=0.027$; asymptotic $\mathrm{p}=0.071 ; \mathrm{p}=0.104$ after Sidak correction for multiple testing). There was a significant increase in the frequency of the HLA-DQA1*0102 allele among AAA cases (67/322 alleles, 20.8\%) compared to controls (44/356 alleles, $12.4 \%$ ) in the Belgian 
Table 3: HLA-DRB I allele frequencies in AAA cases and controls

\begin{tabular}{|c|c|c|c|c|c|c|c|c|c|c|c|c|}
\hline \multirow[b]{3}{*}{ DRBI alleles ${ }^{\mathrm{a}}$} & \multicolumn{6}{|c|}{ AAA Cases } & \multicolumn{6}{|c|}{ Controls } \\
\hline & \multicolumn{2}{|c|}{ Male } & \multicolumn{2}{|c|}{ Female } & \multicolumn{2}{|c|}{ Total } & \multicolumn{2}{|c|}{ Male } & \multicolumn{2}{|c|}{ Female } & \multicolumn{2}{|c|}{ Total } \\
\hline & $\mathrm{n}^{\mathrm{b}}$ & $\%$ & $n^{b}$ & $\%$ & $n^{b}$ & $\%$ & $n^{b}$ & $\%$ & $n^{b}$ & $\%$ & $n^{b}$ & $\%$ \\
\hline \multicolumn{13}{|l|}{ Belgian } \\
\hline *010I & 29 & 9.1 & 3 & 8.3 & 32 & 9.0 & 39 & 10.9 & 14 & 8.3 & 53 & 10.1 \\
\hline$* 0102$ & 7 & 2.2 & I & 2.8 & 8 & 2.2 & 7 & 2.0 & 4 & 2.4 & 11 & 2.1 \\
\hline$* 0103$ & I & 0.3 & I & 2.8 & 2 & 0.6 & 0 & 0 & 0 & 0 & 0 & 0 \\
\hline *030I/0303 & 5 & 1.6 & I & 2.8 & 6 & 1.7 & 7 & 2.0 & 9 & 5.4 & 16 & 3.0 \\
\hline$* 0302$ & 22 & 6.9 & 2 & 5.6 & 24 & 6.7 & 27 & 7.5 & 8 & 4.8 & 35 & 6.7 \\
\hline$*_{0401}$ & 11 & 3.4 & 3 & 8.3 & 14 & 3.9 & 20 & 5.6 & 10 & 6.0 & 30 & 5.7 \\
\hline$* 0402$ & 1 & 0.3 & 0 & 0 & I & 0.3 & 1 & 0.3 & 2 & 1.2 & 3 & 0.6 \\
\hline$* 0403$ & 7 & 2.2 & 2 & 5.6 & 9 & 2.5 & 13 & 3.6 & 5 & 3.0 & 18 & 3.4 \\
\hline$* 0404$ & 9 & 2.8 & 0 & 0 & 9 & 2.5 & 10 & 2.8 & 6 & 3.6 & 16 & 3.0 \\
\hline *0405 & 0 & 0 & 0 & 0 & 0 & 0 & 4 & I.I & 0 & 0 & 4 & 0.8 \\
\hline$* 0407$ & 2 & 0.6 & 0 & 0 & 2 & 0.6 & 2 & 0.6 & 1 & 0.6 & 3 & 0.6 \\
\hline$* 0408$ & 0 & 0 & 0 & 0 & 0 & 0 & I & 0.3 & 0 & 0 & 1 & 0.2 \\
\hline$* 0410$ & I & 0.3 & 0 & 0 & I & 0.3 & 0 & 0 & 0 & 0 & 0 & 0 \\
\hline$* 04 \mid 3$ & 3 & 0.9 & 0 & 0 & 3 & 0.8 & I & 0.3 & I & 0.6 & 2 & 0.4 \\
\hline *070I/0702 & 54 & 16.9 & 3 & 8.3 & 57 & 16.0 & 51 & 14.2 & 18 & 10.7 & 69 & 13.1 \\
\hline$* 0801$ & 9 & 2.8 & 3 & 8.3 & 12 & 3.4 & 11 & 3.1 & 6 & 3.6 & 17 & 3.2 \\
\hline *0802 & 0 & 0 & 0 & 0 & 0 & 0 & 1 & 0.3 & 2 & 1.2 & 3 & 0.6 \\
\hline$* 0803$ & I & 0.3 & 0 & 0 & I & 0.3 & 0 & 0 & 2 & 1.2 & 2 & 0.4 \\
\hline$* 0805$ & 0 & 0 & 0 & 0 & 0 & 0 & 0 & 0 & 0 & 0 & 0 & 0 \\
\hline$* 0901$ & 4 & 1.3 & 0 & 0 & 4 & I.I & 5 & 1.4 & 2 & 1.2 & 7 & 1.3 \\
\hline$* 1001$ & 3 & 0.9 & 0 & 0 & 3 & 0.8 & 7 & 2.0 & 0 & 0 & 7 & 1.3 \\
\hline$* \mid 101$ & 41 & 12.8 & 8 & 22.2 & 49 & 13.8 & 38 & 10.6 & 25 & 14.9 & 63 & 12.0 \\
\hline$* 1102$ & 0 & 0 & 0 & 0 & 0 & 0 & 2 & 0.6 & 1 & 0.6 & 3 & 0.6 \\
\hline$* 1103$ & 7 & 2.2 & I & 2.8 & 8 & 2.2 & 4 & I.I & I & 0.6 & 5 & 1.0 \\
\hline$* 1104$ & I & 0.3 & 0 & 0 & I & 0.3 & I & 0.3 & 0 & 0 & 1 & 0.2 \\
\hline$*|20|$ & 3 & 0.9 & I & 2.8 & 4 & I.I & 6 & 1.7 & 0 & 0 & 6 & I.I \\
\hline$*|30|$ & 14 & 4.4 & 3 & 8.3 & 17 & 4.8 & 10 & 2.8 & 1 & 0.6 & 11 & 2.1 \\
\hline$* 1302$ & 18 & 5.6 & I & 2.8 & 19 & 5.3 & 34 & 9.5 & 13 & 7.7 & 47 & 8.9 \\
\hline$* 1303$ & 3 & 0.9 & 0 & 0 & 3 & 0.8 & 3 & 0.8 & 4 & 2.4 & 7 & 1.3 \\
\hline$* 1305$ & 0 & 0 & 0 & 0 & 0 & 0 & 0 & 0 & 0 & 0 & 0 & 0 \\
\hline$*|40|$ & 5 & 1.6 & 0 & 0 & 5 & 1.4 & 5 & 1.4 & I & 0.6 & 6 & 1.1 \\
\hline$* 1407$ & 3 & 0.9 & 0 & 0 & 3 & 0.8 & 6 & 1.7 & 3 & 1.8 & 9 & 1.7 \\
\hline$*|50|$ & 0 & 0 & 0 & 0 & 0 & 0 & I & 0.3 & 2 & 1.2 & 3 & 0.6 \\
\hline$* 1502$ & 1 & 0.3 & 0 & 0 & I & 0.3 & 0 & 0 & 0 & 0 & 0 & 0 \\
\hline$* 1601$ & 47 & 14.7 & 2 & 5.6 & 49 & 13.8 & 27 & 7.5 & 19 & 11.3 & 46 & 8.7 \\
\hline$* 1602$ & 0 & 0 & 0 & 0 & 0 & 0 & 2 & 0.6 & 0 & 0 & 2 & 0.4 \\
\hline$* 0301 / 0303$ or $* 0302$ & 1 & 0.3 & 0 & 0 & I & 0.3 & 2 & 0.6 & 3 & 1.8 & 5 & 1.0 \\
\hline$* 0403$ or $* 0407$ & 0 & 0 & 0 & 0 & 0 & 0 & I & 0.3 & I & 0.6 & 2 & 0.4 \\
\hline$* 0404$ or $* 0408$ & 0 & 0 & 0 & 0 & 0 & 0 & 0 & 0 & I & 0.6 & 1 & 0.2 \\
\hline$* 1101$ or $* 1104$ & 0 & 0 & 0 & 0 & 0 & 0 & 0 & 0 & 0 & 0 & 0 & 0 \\
\hline$* 1301$ or $* 1302$ & 7 & 2.2 & I & 2.8 & 8 & 2.2 & 5 & 1.4 & 2 & 1.2 & 7 & 1.3 \\
\hline$* 1401$ or $* 1407$ & 0 & 0 & 0 & 0 & 0 & 0 & 2 & 0.6 & I & 0.6 & 3 & 0.6 \\
\hline CND & 0 & 0 & 0 & 0 & 0 & 0 & 2 & 0.6 & 0 & 0 & 2 & 0.4 \\
\hline
\end{tabular}


Table 3: HLA-DRB I allele frequencies in AAA cases and controls (Continued)

\begin{tabular}{|c|c|c|c|c|c|c|c|c|c|c|c|c|}
\hline Total & 320 & 100.0 & 36 & 100.0 & 356 & 100.0 & 358 & 100.0 & 168 & 100.0 & 526 & 100.0 \\
\hline \multicolumn{13}{|l|}{ Canadian } \\
\hline *0I0I & 27 & 8.8 & 7 & 7.0 & 34 & 8.3 & 4 & 6.1 & 21 & 8.5 & 25 & 8.0 \\
\hline$* 0102$ & 1 & 0.3 & I & 1.0 & 2 & 0.5 & 2 & 3.0 & 4 & 1.6 & 6 & 1.9 \\
\hline$* 0103$ & 6 & 1.9 & 1 & 1.0 & 7 & 1.7 & 2 & 3.0 & 4 & 1.6 & 6 & 1.9 \\
\hline *0301/0303 & 13 & 4.2 & 2 & 2.0 & 15 & 3.7 & 3 & 4.5 & 7 & 2.8 & 10 & 3.2 \\
\hline *0302 & 26 & 8.4 & 12 & 12 & 38 & 9.3 & 2 & 3.0 & 23 & 9.3 & 25 & 8.0 \\
\hline *040 I & 31 & 10.0 & 8 & 8.0 & 39 & 9.6 & 6 & 9.1 & 28 & 11.3 & 34 & 10.8 \\
\hline *0402 & 2 & 0.6 & 0 & 0 & 2 & 0.5 & 0 & 0 & 0 & 0 & 0 & 0 \\
\hline *0403 & 8 & 2.6 & 7 & 7.0 & 15 & 3.7 & 3 & 4.5 & 7 & 2.8 & 10 & 3.2 \\
\hline$* 0404$ & 17 & 5.5 & 4 & 4.0 & 21 & 5.1 & 3 & 4.5 & II & 4.4 & 14 & 4.5 \\
\hline *0405 & 0 & 0 & 0 & 0 & 0 & 0 & 0 & 0 & 2 & 0.8 & 2 & 0.6 \\
\hline *0407 & 1 & 0.3 & 2 & 2.0 & 3 & 0.7 & 0 & 0 & 0 & 0 & 0 & 0 \\
\hline *0408 & 1 & 0.3 & 0 & 0 & 1 & 0.2 & 0 & 0 & 0 & 0 & 0 & 0 \\
\hline$* 0410$ & 0 & 0 & 0 & 0 & 0 & 0 & 0 & 0 & 0 & 0 & 0 & 0 \\
\hline$* 0413$ & 0 & 0 & 0 & 0 & 0 & 0 & 0 & 0 & 0 & 0 & 0 & 0 \\
\hline *070I/0702 & 49 & 15.9 & 18 & 18 & 67 & 16.4 & 18 & 27.3 & 36 & 14.5 & 54 & 17.2 \\
\hline *080I & 7 & 2.3 & 1 & 1.0 & 8 & 2.0 & 0 & 0 & 6 & 2.4 & 6 & 1.9 \\
\hline *0802 & 0 & 0 & I & 1.0 & 1 & 0.2 & 0 & 0 & 2 & 0.8 & 2 & 0.6 \\
\hline *0803 & 0 & 0 & 0 & 0 & 0 & 0 & 0 & 0 & I & 0.4 & 1 & 0.3 \\
\hline *0805 & 0 & 0 & 0 & 0 & 0 & 0 & 1 & 1.5 & 0 & 0 & 1 & 0.3 \\
\hline *0901 & 7 & 2.3 & 0 & 0 & 7 & 1.7 & 0 & 0 & 2 & 0.8 & 2 & 0.6 \\
\hline$* 1001$ & 3 & 1.0 & 0 & 0 & 3 & 0.7 & 0 & 0 & 3 & 1.2 & 3 & 1.0 \\
\hline$* 1101$ & 22 & 7.1 & 7 & 7.0 & 29 & 7.1 & 3 & 4.5 & 21 & 8.5 & 24 & 7.6 \\
\hline$* 1102$ & 1 & 0.3 & 0 & 0 & 1 & 0.2 & 0 & 0 & $\mathrm{I}$ & 0.4 & 1 & 0.3 \\
\hline$* 1103$ & 1 & 0.3 & I & 1.0 & 2 & 0.5 & 0 & 0 & 0 & 0 & 0 & 0 \\
\hline *1। 04 & 0 & 0 & 0 & 0 & 0 & 0 & 0 & 0 & 0 & 0 & 0 & 0 \\
\hline$*|20|$ & 0 & 0 & 1 & 1.0 & 1 & 0.2 & 0 & 0 & 3 & 1.2 & 3 & 1.0 \\
\hline$*|30|$ & 8 & 2.6 & 3 & 3.0 & 11 & 2.7 & 1 & 1.5 & 1 & 0.4 & 2 & 0.6 \\
\hline$* 1302$ & 12 & 3.9 & 8 & 8.0 & 20 & 4.9 & 3 & 4.5 & 21 & 8.5 & 24 & 7.6 \\
\hline$* 1303$ & 3 & 1.0 & 0 & 0 & 3 & 0.7 & 0 & 0 & 0 & 0 & 0 & 0 \\
\hline$* 1305$ & 1 & 0.3 & 0 & 0 & 1 & 0.2 & 0 & 0 & 1 & 0.4 & I & 0.3 \\
\hline$*|40|$ & 5 & 1.6 & 0 & 0 & 5 & 1.2 & 2 & 3.0 & 2 & 0.8 & 4 & 1.3 \\
\hline *I407 & 2 & 0.6 & 0 & 0 & 2 & 0.5 & 1 & 1.5 & 6 & 2.4 & 7 & 2.2 \\
\hline$*|50|$ & 0 & 0 & 0 & 0 & 0 & 0 & 0 & 0 & 0 & 0 & 0 & 0 \\
\hline *1502 & 0 & 0 & 0 & 0 & 0 & 0 & 0 & 0 & 0 & 0 & 0 & 0 \\
\hline$*|60|$ & 42 & 13.6 & 15 & 15 & 57 & 14.0 & 9 & 13.6 & 29 & 11.7 & 38 & 12.1 \\
\hline$* 1602$ & 0 & 0 & 0 & 0 & 0 & 0 & 0 & 0 & 0 & 0 & 0 & 0 \\
\hline$* 0301 / 0303$ or $* 0302$ & 4 & 1.3 & 0 & 0 & 4 & 1.0 & 1 & 1.5 & 2 & 0.8 & 3 & 1.0 \\
\hline$* 0403$ or $* 0407$ & 1 & 0.3 & 0 & 0 & 1 & 0.2 & 0 & 0 & 0 & 0 & 0 & 0 \\
\hline *0404 or *0408 & 0 & 0 & 1 & 1.0 & 1 & 0.2 & 0 & 0 & 0 & 0 & 0 & 0 \\
\hline *1101 or *1104 & 1 & 0.3 & 0 & 0 & 1 & 0.2 & 0 & 0 & 0 & 0 & 0 & 0 \\
\hline$* 1301$ or *1302 & 4 & 1.3 & 0 & 0 & 4 & 1.0 & 1 & 1.5 & 1 & 0.4 & 2 & 0.6 \\
\hline$* 1401$ or $* 1407$ & 1 & 0.3 & 0 & 0 & 1 & 0.2 & 1 & 1.5 & 2 & 0.8 & 3 & 1.0 \\
\hline CND & 1 & 0.3 & 0 & 0 & 1 & 0.2 & 0 & 0 & 1 & 0.4 & 1 & 0.3 \\
\hline Total & 308 & 100.0 & 100 & 100.0 & 408 & 100.0 & 66 & 100.0 & 248 & 100.0 & 314 & 100.0 \\
\hline
\end{tabular}

AAA: abdominal aortic aneurysm; CND: could not be determined.

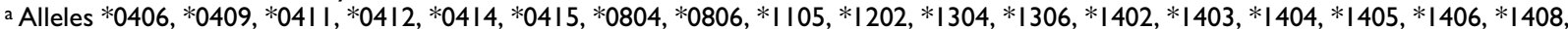

$* 1409, * 1410$ and *1503 were also assayed but were not present in either population.

b Number of alleles.

c Could be classified into two possible alleles, but could not be classified into one particular allele. 
Table 4: HLA-DRB3-5 allele frequencies in AAA cases and controls

\begin{tabular}{|c|c|c|c|c|c|c|c|c|c|c|c|c|}
\hline \multirow[b]{3}{*}{$\begin{array}{l}\text { DRB3-5 } \\
\text { alleles }\end{array}$} & \multicolumn{6}{|c|}{ AAA Cases } & \multicolumn{6}{|c|}{ Controls } \\
\hline & \multicolumn{2}{|c|}{ Male } & \multicolumn{2}{|c|}{ Female } & \multicolumn{2}{|c|}{ Total } & \multicolumn{2}{|c|}{ Male } & \multicolumn{2}{|c|}{ Female } & \multicolumn{2}{|c|}{ Total } \\
\hline & $\mathrm{n}^{\mathrm{a}}$ & $\%$ & $\mathrm{n}^{\mathrm{a}}$ & $\%$ & $\mathrm{n}^{\mathrm{a}}$ & $\%$ & $\mathrm{n}^{\mathrm{a}}$ & $\%$ & $\mathrm{n}^{\mathrm{a}}$ & $\%$ & $\mathrm{n}^{\mathrm{a}}$ & $\%$ \\
\hline \multicolumn{13}{|l|}{ Belgian } \\
\hline B3*0I0I & 40 & 12.5 & 8 & 22.2 & 48 & 13.5 & 53 & 14.8 & 24 & 14.4 & 77 & 14.6 \\
\hline B3*020I & 11 & 3.4 & I & 2.8 & 12 & 3.4 & 11 & 3.1 & 0 & 0 & II & 2.1 \\
\hline B3*0202 & 63 & 19.7 & 8 & 22.2 & 71 & 19.9 & 86 & 24.0 & 35 & 21.0 & 121 & 23.0 \\
\hline B3*030I & 34 & 10.6 & 7 & 19.4 & 41 & 11.5 & 45 & 12.5 & 22 & 13.2 & 67 & 12.7 \\
\hline $\mathrm{B} 4 * 0 \mathrm{IOI}$ & 94 & 29.4 & 7 & 19.4 & 101 & 28.4 & 103 & 28.7 & 52 & 31.1 & 155 & 29.5 \\
\hline B5*0I0I & 50 & 15.6 & 2 & 5.6 & 52 & 14.6 & 31 & 8.6 & 16 & 9.6 & 47 & 8.9 \\
\hline $\mathrm{B} 5 * 0102$ & 4 & 1.3 & 0 & 0 & 4 & I.I & 2 & 0.6 & 3 & 1.8 & 5 & 1.0 \\
\hline $\mathrm{B} 5 * 020 \mathrm{I}$ & 7 & 2.2 & 0 & 0 & 7 & 2.0 & 6 & 1.7 & I & 0.6 & 7 & 1.3 \\
\hline $\mathrm{B} 5 * 0203$ & 0 & 0 & 0 & 0 & 0 & 0.0 & 0 & 0 & 0 & 0 & 0 & 0 \\
\hline $\begin{array}{l}\mathrm{B} 3 * 020 \mathrm{I} \\
\text { or } * 0202 \\
\mathrm{~b}\end{array}$ & 13 & 4.1 & I & 2.8 & 14 & 3.9 & 20 & 5.6 & 10 & 6.0 & 30 & 5.7 \\
\hline CND & 4 & 1.3 & 2 & 5.6 & 6 & 1.7 & 2 & 0.6 & 4 & 2.4 & 6 & I.I \\
\hline Total & 320 & 100.0 & 36 & 100.0 & 356 & 100.0 & 359 & 100.0 & 167 & 100.0 & 526 & 100.0 \\
\hline \multicolumn{13}{|l|}{ Canadian } \\
\hline B3*0।0I & 53 & 17.2 & 17 & 17 & 70 & 17.2 & 9 & 13.6 & 38 & 15.3 & 47 & 15.0 \\
\hline B3*020I & 9 & 2.9 & 5 & 5.0 & 14 & 3.4 & 0 & 0 & 6 & 2.4 & 6 & 1.9 \\
\hline $\mathrm{B} 3 * 0202$ & 44 & 14.3 & 15 & 15 & 59 & 14.5 & 8 & 12.1 & 46 & 18.5 & 54 & 17.2 \\
\hline B3*030I & 38 & 12.3 & 13 & 13 & 51 & 12.5 & 11 & 16.7 & 32 & 12.9 & 43 & 13.7 \\
\hline B4*0I0I & 108 & 35.1 & 32 & 32 & 140 & 34.3 & 26 & 39.4 & 80 & 32.3 & 106 & 33.8 \\
\hline B5*0I0I & 45 & 14.6 & 14 & 14 & 59 & 14.5 & 12 & 18.2 & 38 & 15.3 & 50 & 15.9 \\
\hline $\mathrm{B} 5 * 0102$ & 1 & 0.3 & 1 & 1.0 & 2 & 0.5 & 0 & 0 & 3 & 1.2 & 3 & 1.0 \\
\hline $\mathrm{B} 5 * 020 \mathrm{I}$ & 2 & 0.6 & I & 1.0 & 3 & 0.7 & 0 & 0 & I & 0.4 & 1 & 0.3 \\
\hline B5*0203 & 1 & 0.3 & 0 & 0 & 1 & 0.2 & 0 & 0 & 0 & 0 & 0 & 0 \\
\hline $\begin{array}{l}\mathrm{B} 3 * 0201 \\
\text { or } * 0202 \\
\mathrm{~b}\end{array}$ & 3 & 1.0 & 0 & 0 & 3 & 0.7 & 0 & 0 & 0 & 0 & 0 & 0 \\
\hline CND & 4 & 1.3 & 2 & 2.0 & 6 & 1.5 & 0 & 0 & 4 & 1.6 & 4 & 1.3 \\
\hline Total & 308 & 100.0 & 100 & 100.0 & 408 & 100.0 & 66 & 100.0 & 248 & 100.0 & 314 & 100.0 \\
\hline
\end{tabular}

AAA: abdominal aortic aneurysm; CND: could not be determined.

a Number of alleles.

b Could be classified into HLA-DRB*020I or *0202, but could not be classified into one particular allele.

male population (empirical $\mathrm{p}=0.019$; asymptotic $\mathrm{p}=$ 0.003 ). The odds ratio was 1.86 and $95 \%$ confidence interval was 1.56 to 2.22 for the HLA-DQA $1 * 0102$ allele versus all the other alleles in the Belgian males. Since the number of female cases was small, no conclusions can be drawn about the association in females. In the Belgian population, the association between AAA and DQA1 was greater among those with a family history of AAA (empirical $\mathrm{p}=0.069$; asymptotic $\mathrm{p}=0.067$ ) compared to those without a family history of AAA (empirical $\mathrm{p}=0.157$; asymptotic $p=0.164)$. Since the proportion of males was similar in the group with (88\%) and without $(90 \%)$ family history, the result was not due to difference in the number of males in each group. No additional significant associations were found between AAA cases and controls when AAA cases were stratified by family history of AAA (Table 6).

The results of haplotype analyses for the HLA-DQA1, DQB1, -DRB1 and -DRB3-5 loci between AAA cases and controls are shown in Tables 7 and 8. Only the most significant haplotypes and their p-values when analyzing two-locus haplotypes are listed. Although most of the frequencies of the most significant haplotypes were relatively rare $(<5 \%)$, haplotype DQA $1 * 0102 / \mathrm{DRB} 1 * 1601$ had a relatively high frequency (11.6\%; Table 7). Using the global score statistics, the nominal p-value was 0.049 and with haplotype-specific score statistics, the nominal pvalue was 0.002 for this haplotype, which is not as strong statistical evidence as the result for the DQA1 locus alone. 
Table 5: Empirical and asymptotic p-values for comparisons between AAA cases and controls within each population and stratified by sex

\begin{tabular}{|c|c|c|c|c|c|c|c|c|c|c|c|c|}
\hline \multirow[t]{3}{*}{ Locus } & \multicolumn{6}{|c|}{ Belgian } & \multicolumn{6}{|c|}{ Canadian } \\
\hline & \multicolumn{2}{|c|}{ Male $^{\mathrm{a}}$} & \multicolumn{2}{|c|}{ Female $^{\mathrm{a}}$} & \multicolumn{2}{|c|}{ Total } & \multicolumn{2}{|c|}{ Male $^{\mathrm{a}}$} & \multicolumn{2}{|c|}{ Female $^{\mathrm{a}}$} & \multicolumn{2}{|c|}{ Total } \\
\hline & $\mathrm{EPb}$ & $\mathrm{APc}$ & $\mathrm{EPb}$ & $A P^{c}$ & $\mathrm{EPb}$ & $A P^{c}$ & $\mathrm{EPb}$ & $\mathrm{APc}$ & $\mathrm{EPb}$ & $A P^{c}$ & $\mathrm{EPb}$ & $A P^{c}$ \\
\hline DQAI & 0.027 & 0.071 & 0.899 & 0.900 & 0.039 & 0.049 & 0.392 & 0.487 & 0.340 & 0.443 & 0.589 & 0.682 \\
\hline DQBI & 0.083 & 0.130 & 0.495 & 0.710 & 0.129 & 0.144 & 0.917 & 0.972 & 0.405 & 0.553 & 0.371 & 0.510 \\
\hline DRBI & 0.252 & 0.375 & 0.218 & 0.653 & 0.114 & 0.200 & 0.465 & 0.890 & 0.408 & 0.864 & 0.246 & 0.545 \\
\hline DRB3-5 & 0.145 & 0.217 & 0.239 & 0.308 & 0.217 & 0.308 & 0.745 & 0.778 & 0.926 & 0.952 & 0.766 & 0.739 \\
\hline
\end{tabular}

AAA: abdominal aortic aneurysm; EP: empirical p-value; AP: asymptotic p-value.

a Number of Belgian AAA cases: Male = 16I, Female = 19; Canadian AAA cases: Male = 155, Female = 52; Belgian controls: Male = I78, Female = 91; Canadian controls: Male = 39, Female = 118.

b Global empirical p-values were calculated by permutation test using the CLUMP [28] program.

c Asymptotic p-values were calculated by $\chi^{2}$ test.

In addition, the haplotype results were not significant among the Belgian males (Table 8), as was observed for DQA1 alone.

\section{Discussion}

AAA is a relatively common disease in that an estimated $1-6 \%$ of the population in industrialized countries harbor aneurysms [32]. Rupture of AAAs causes 1-2\% of all deaths in males over 65 years of age in Western countries $[33,34]$. It has been suggested that AAAs are a complex disease $[35,36]$. Two segregation studies favored a genetic model in explaining the familial aggregation of AAA and suggested the presence of a major gene effect $[35,36]$. Recently, we reported on a collection of 233 families with at least two individuals affected with AAA [37], and identified two genetic susceptibility loci for AAA on chromosomes $19 q 13$ and 4q31 [38]. We also recently reported a genetic association study for polymorphisms in biologically relevant candidate genes for AAA, and found evidence for an association between tissue inhibitor of metalloproteinases 1 (TIMP1) polymorphisms and AAA [31].
It is well known that there are large differences in the frequencies of the HLA polymorphisms in different populations. We, therefore, carried out the analyses with Belgian and Canadian populations separately, to avoid potential false positive results because of population stratification. There have, however, been several examples where substructure within Caucasian samples has been explored and identified $[39,40]$, thus the possibility of cryptic substructure within each population remains a concern. Although ancestry informative marker (AIM) panels exist, these panels have been created with the intent of distinguishing the major racial/ethnic groups, and may not be sensitive for detecting population substructure within a single racial/ethnic group. Ideally, it will be possible in the future to utilize AIM panels that are specific to addressing the question of cryptic relatedness within the Caucasian population.

We observed a potential association between the HLADQA1 locus and AAA among the Belgian males. In particular, there was a statistically significant difference in the frequency of the HLA-DQA $1 * 0102$ allele between AAA

Table 6: Analysis of HLA loci in AAA cases and controls when stratified by family history of AAA

\begin{tabular}{|c|c|c|c|c|c|c|c|c|}
\hline \multirow[t]{3}{*}{ Locus } & \multicolumn{4}{|c|}{ Belgian } & \multicolumn{4}{|c|}{ Canadian } \\
\hline & \multicolumn{2}{|c|}{ Family history } & \multicolumn{2}{|c|}{ No family history } & \multicolumn{2}{|c|}{ Family history } & \multicolumn{2}{|c|}{ No family history } \\
\hline & $\mathrm{EPa}$ & $\mathrm{APb}$ & $\mathrm{EPa}$ & $\mathrm{APb}$ & $\mathrm{EPa}$ & $\mathrm{APb}$ & $\mathrm{EPa}$ & $\mathrm{APb}$ \\
\hline DQAI & 0.069 & 0.067 & 0.157 & 0.164 & 0.198 & 0.284 & 0.764 & 0.832 \\
\hline DQBI & 0.419 & 0.415 & 0.305 & 0.317 & 0.384 & 0.532 & 0.432 & 0.571 \\
\hline DRBI & 0.597 & 0.811 & 0.099 & 0.171 & 0.232 & 0.545 & 0.242 & 0.659 \\
\hline DRB3-5 & 0.356 & 0.454 & 0.314 & 0.414 & 0.831 & 0.796 & 0.490 & 0.581 \\
\hline
\end{tabular}

AAA: abdominal aortic aneurysm; EP: empirical p-value; AP: asymptotic $p$-value.

a Global empirical p-values were calculated by permutation test using the CLUMP [28] program.

b Asymptotic $p$-values were calculated by $\chi^{2}$ test. 
Table 7: Haplotype analyses when comparing two HLA loci

\begin{tabular}{|c|c|c|c|c|c|c|c|}
\hline \multirow{2}{*}{$\begin{array}{l}\text { Haplotype } \\
\text { combination }\end{array}$} & \multirow{2}{*}{$\begin{array}{l}\text { Global Score } \\
\text { Statistics }\end{array}$} & \multirow[t]{2}{*}{ DF } & \multirow[t]{2}{*}{$P$ value } & \multicolumn{2}{|c|}{ Most significant haplotype } & \multirow{2}{*}{$\begin{array}{l}\text { Haplotype- } \\
\text { specific score } \\
\text { statistics }^{\mathrm{a}}\end{array}$} & \multirow[t]{2}{*}{$P$ value } \\
\hline & & & & Haplotype & $\begin{array}{c}\text { Population } \\
\text { Frequency (\%) }\end{array}$ & & \\
\hline DQAI+DQBI & 62.7 & 58 & 0.311 & $020 \mathrm{I} \sim 0302$ & 1.2 & 3.01 & 0.003 \\
\hline DQAI+DRBI & 91.8 & 71 & 0.049 & $0102 \sim 1601$ & 11.6 & 3.04 & 0.002 \\
\hline DQAI+DRB3-5 & 50.6 & 51 & 0.491 & $0101 \sim B 3-0202$ & 4.8 & -2.39 & 0.017 \\
\hline DQBI+DRBI & 125.7 & 119 & 0.319 & $0604 \sim 1301$ & 1.1 & 2.36 & 0.018 \\
\hline DQBI+DRB3-5 & 66.2 & 77 & 0.806 & 030I B3-020 I & 1.9 & 2.02 & 0.044 \\
\hline DRBI+DRB3-5 & 115.1 & 114 & 0.453 & $|60| \sim B 5-010 \mid$ & 10.3 & 2.35 & 0.019 \\
\hline
\end{tabular}

DF: degrees of freedom; AAA: abdominal aortic aneurysm.

a Haplo.stats program [29] was used for the analysis. Rare haplotypes with frequencies of $\leq 0.01$ were pooled into a single baseline group. Results on haplotypes with only two loci are reported.

Table 8: Haplotype analyses for two HLA loci combined and stratified by population and sex

\begin{tabular}{|c|c|c|c|c|c|c|c|c|c|}
\hline \multirow[t]{2}{*}{ Population } & \multirow[t]{2}{*}{ Sex } & \multirow{2}{*}{$\begin{array}{l}\text { Haplotype } \\
\text { combination }\end{array}$} & \multirow{2}{*}{$\begin{array}{l}\text { Global Score } \\
\text { Statistics }\end{array}$} & \multirow[t]{2}{*}{ DF } & \multirow[t]{2}{*}{$P$ value } & \multicolumn{2}{|c|}{ Most significant haplotype } & \multirow{2}{*}{$\begin{array}{l}\text { Haplotype-specific } \\
\text { score statistics }^{\mathrm{a}}\end{array}$} & \multirow[t]{2}{*}{$P$ value $^{a}$} \\
\hline & & & & & & Haplotype & $\begin{array}{c}\text { Population } \\
\text { frequency (\%) }\end{array}$ & & \\
\hline \multicolumn{10}{|l|}{ Belgian } \\
\hline & Male & DQAI+DQBI & 54.0 & 42 & 0.102 & $0102 \sim 0602$ & 9.4 & 2.97 & 0.003 \\
\hline & & DQAI+DRBI & 57.8 & 53 & 0.303 & $0102 \sim 1601$ & 10.5 & 3.50 & 0.001 \\
\hline & & DQAI+DRB3-5 & 44.8 & 39 & 0.240 & 0I02 B5-0IOI & 9.4 & 2.95 & 0.003 \\
\hline & & DQBI+DRBI & 81.1 & 82 & 0.508 & $0602 \sim 1601$ & 8.9 & 2.88 & 0.004 \\
\hline & & DQBI+DRB3-5 & 53.5 & 60 & 0.710 & 0602 B5-0I0I & 9.2 & 2.88 & 0.004 \\
\hline & & DRBI+DRB3-5 & 84.5 & 81 & 0.374 & I60I B5-0 01 & 9.3 & 3.24 & 0.001 \\
\hline & Female & DQAI+DQBI & 29.7 & 28 & 0.380 & $0102 \sim 0603$ & 1.3 & 3.48 & 0.001 \\
\hline & & DQAI+DRBI & 41.6 & 34 & 0.173 & $0102 \sim 1301$ & 2.1 & 3.52 & 0.001 \\
\hline & & DQAI+DRB3-5 & 22.9 & 26 & 0.638 & $050 \mathrm{I} \sim \mathrm{B} 3-020 \mathrm{I}$ & 0.6 & 2.12 & 0.033 \\
\hline & & DQBI+DRBI & 40.7 & 49 & 0.794 & $0604 \sim 1301$ & 2.2 & 2.81 & 0.005 \\
\hline & & DQBI+DRB3-5 & 38.0 & 37 & 0.422 & 0603 B5-0IOI & 0.6 & 2.74 & 0.006 \\
\hline & & DRBI+DRB3-5 & 49.4 & 48 & 0.418 & $|30| \sim B 3-030 \mid$ & 2.2 & 2.92 & 0.003 \\
\hline \multicolumn{10}{|l|}{ Canadian } \\
\hline & Male & DQAI+DQBI & 27.6 & 32 & 0.691 & $0 \mathrm{I} 0 \mathrm{I} \sim 020 \mathrm{I}$ & 0.3 & -2.16 & 0.031 \\
\hline & & DQAI+DRBI & 36.8 & 38 & 0.527 & 0101 0102 & 0.8 & -2.34 & 0.020 \\
\hline & & DQAI+DRB3-5 & 27.8 & 33 & 0.724 & 040I B5-0 I $0 \mathrm{I}$ & 0.3 & -2.00 & 0.046 \\
\hline & & DQBI+DRBI & 55.7 & 56 & 0.486 & $050 \mathrm{I} \sim 0 \mathrm{I} 02$ & 0.8 & -2.22 & 0.027 \\
\hline & & DQBI+DRB3-5 & 36.3 & 49 & 0.910 & 03032 B3-030 I & I.I & -3.32 & 0.001 \\
\hline & & DRBI+DRB3-5 & 55.6 & 63 & 0.735 & 070I B4-0I0I & 11.4 & -2.19 & 0.028 \\
\hline & Female & DQAI+DQBI & 29.1 & 31 & 0.563 & $020 \mathrm{I} \sim 0302$ & 1.2 & 2.01 & 0.045 \\
\hline & & DQAI+DRBI & 41.4 & 38 & 0.326 & $0103 \sim 1301$ & 0.4 & 2.25 & 0.024 \\
\hline & & DQAI+DRB3-5 & 34.1 & 33 & 0.414 & $0102 \sim B 3-0101$ & 0.6 & 2.37 & 0.018 \\
\hline & & DQBI+DRBI & 69.6 & 62 & 0.237 & 0302 0407 & 0.5 & 2.71 & 0.007 \\
\hline & & DQBI+DRB3-5 & 49.0 & 43 & 0.245 & 0602 B4-0IOI & 0.9 & 2.93 & 0.003 \\
\hline & & DRBI+DRB3-5 & 61.1 & 60 & 0.437 & $030 \mathrm{I} \sim \mathrm{B} 3-030 \mathrm{I}$ & 0.2 & 2.37 & 0.017 \\
\hline
\end{tabular}

DF: degrees of freedom; AAA: abdominal aortic aneurysm.

a Haplo.stats program [29] was used for the analyses. Rare haplotype with frequencies $\leq 0.01$ were pooled into a single baseline group. Results on haplotypes with only two loci are reported. 
cases and controls in the Belgian males. This finding suggests that the HLA-DQA1 locus harbors a genetic risk factor for AAAs. Since the number of the Belgian female cases was small $(\mathrm{n}=20)$, further work with a larger sample will be needed to investigate the role of HLA in female AAA cases. No significant associations were observed in the Canadian population, although the frequency of the DQA1*0102 allele was also higher in the Canadian cases than the controls.

Several association studies between HLA polymorphisms and AAA have been performed previously. Most of the previous studies were small and different methods to type the HLA alleles were used making it difficult to compare these studies to each other (Table 9) [11-19]. The most common finding was an association between AAA and HLA-DRB $1 * 02$ alleles, which are divided into -DRB1*15 and $* 16$ alleles. Tilson et al [12] demonstrated this association in 26 African-Americans. Rasmussen et al [13,16,17] reported a similar result in 37 North Americans and Hirose et al [14] reported this association in 46 Japanese. Sugimoto et al [18] $(\mathrm{n}=49)$ and Moňux et al [19] $(\mathrm{n}=$ 72), however, failed to find such an association in their studies. Our study showed a marginally significant association between haplotype DQA1*0102/DRB1*1601 and AAA. The HLA-DRB1*1601 allele, however, was not significantly associated with AAA by permutation test (empirical $\mathrm{p}=0.253$; asymptotic $\mathrm{p}=0.02$ ). There was no significant association between HLA-DRB1 *04 alleles and AAA in the current study, which differs from the results by Rasmussen et al [13] and Moňux et al [19] when using 37 and 72 AAA cases, respectively. No prior association stud- ies between AAA and HLA-DQA1 locus were performed (Table 9).

The HLA-DQA1*0102 allele, which showed a potential association with AAA in our study, has been found to be associated previously with other diseases. The HLADQA $1 * 0102$ allele is known to be a protective allele against type 1 diabetes mellitus (DM) and systemic lupus erythematosus, which are classical autoimmune diseases $[41,42]$. Interestingly, type $2 \mathrm{DM}$, which is a well-known risk factor for many cardiovascular diseases, is protective against AAA in the U.S. population [23].

Although there is growing evidence of the association between the HLA system and autoimmune diseases, including AAA, the precise disease-causing mechanism is yet to be defined. It is also possible that an association between HLA and disease is only a marker for an undiscovered polymorphism in a linked gene. There are at least 120 additional genes in the MHC region. Most of these additional genes in the HLA class II region are involved in immunological functions that relate to the HLA class I and II genes $[7,43]$. Because of the high density of potentially important genes in the $\mathrm{MHC}$ region, linkage disequilibrium around this region makes it difficult to identify the exact susceptibility gene for a disease.

Limitations of the study were: 1) the statistical power was relatively low (for significance level of 0.05 the power was 0.49 in the Belgian males; 0.14 in the Belgian females; 0.16 in the Canadian males and 0.22 in the Canadian females using the observed allele frequencies), and after correction for multiple testing, none of the results

Table 9: Previous HLA studies on AAA

\begin{tabular}{|c|c|c|c|c|c|}
\hline Study & Country & n (Inflammatory AAA) ${ }^{a}$ & HLA locus studied & Significant allele ${ }^{b}$ & $P$ \\
\hline Norrgård et al I984 [II] & Sweden & 48 & HLA-A, $-\mathrm{B}^{\mathrm{c}}$ & None & $\mathrm{N} / \mathrm{A}$ \\
\hline \multirow{2}{*}{ Tilson et al 1996 [12] } & US & 26 & HLA-DRBI & $\mathrm{DRB} \mid * 02$ & 0.04 \\
\hline & & & & $\mathrm{DRB} \mid * 12$ & 0.02 \\
\hline \multirow[t]{2}{*}{ Rasmussen et al 1997 [13] } & US & 37 & HLA-DQBI, DRBI & DRBI*15 & $<0.05$ \\
\hline & & (37) & & DRBI*0404 & $<0.05$ \\
\hline Hirose et al 1998 [14] & Japan & 46 & HLA-DRc & DR2(I5) & $<0.005$ \\
\hline Hirose \& Tilson 1999 [15] & Japan & 36 & HLA-DQc & DQ3 & 0.014 \\
\hline \multirow[t]{2}{*}{ Rasmussen et al 200। [16] } & US & 142 & HLA-DRBI & DRBI*02 & 0.03 \\
\hline & & $(40)$ & & & $0.01 d$ \\
\hline \multirow[t]{3}{*}{ Rasmussen et al 2002 [17] } & US & 142 & HLA-DRBI & DRBI*02 & $<0.01$ \\
\hline & & & & DRBI*08 & 0.04 \\
\hline & & & & DRBI*I4 & $<0.01$ \\
\hline \multirow[t]{2}{*}{ Sugimoto et al 2003 [18] } & Japan & 49 & HLA-A, -B, -DR & A2 & $<0.05$ \\
\hline & & & & B6I & $<0.005$ \\
\hline Moňux et al 2003 [19] & Spain & 72 & HLA-DRBI & DRB $|* 040|$ & 0.02 \\
\hline
\end{tabular}

AAA: abdominal aortic aneurysm.

a Inflammatory AAA was categorized and analyzed separately.

b Allele which had a significantly different frequency between AAA cases and controls.

c Serological typing was carried out.

d P-value for inflammatory AAA. 
remained significant. Nevertheless, the findings were consistent in the sense that the HLA-DQA1*0102 allele frequency was always higher in the case groups than in the control groups $(20.8 \%$ vs. $12.4 \%$ in the Belgian males; $21 \%$ vs. $16 \%$ in the Belgian females; $18.8 \%$ vs. 16.7 in the Canadian males; and $20.8 \%$ vs. $15 \%$ in the Canadian females); 2) although the overall sample size was not small, the number of alleles was large leading to relatively small counts for each allele; 3 ) we did not test the interaction with other loci and environmental factors, such as age, or smoking; and 4) our sampling scheme including spousal controls could lead to false positive results because of the potential effect of the HLA loci on mate selection. The HLA region has been suggested to influence mate selection based on odor differences coded by the MHC genes, with a preference for dissimilar partners, in both mice and humans [44], although these findings are not always consistent. The presence of negative assortative mating could lead to false positive findings when comparing AAA cases to control spouses.

\section{Conclusion}

We found evidence for genetic association between the HLA-DQA1 locus and AAA suggesting that this genomic region harbors a genetic risk factor for AAAs in the Belgian male population. The same DQA1*0102 allele was present at higher frequency among the Belgian female, Canadian male and Canadian female cases supporting the hypothesis that autoimmunity contributes to the pathogenesis of AAAs. In future studies, the findings need to be assessed in another, larger sample.

\section{Competing interests}

The author(s) declare that they have no competing interests.

\section{Authors' contributions}

TO carried out the molecular assays, scored results and prepared the manuscript. LG designed the study and scored results. KG designed and carried out statistical analyses as well as prepared the manuscript. MS carried out the molecular assays. GT designed the study and analyzed data. WL designed the study and analyzed data. AP carried out statistical analyses. QL carried out statistical analyses. HS designed the study and carried out some of the molecular assays. NS recruited Belgian patients and controls into the study. RL recruited Belgian patients and controls into the study. GM recruited Canadian patients and controls into the study. CA recruited Canadian patients and controls into the study. TS provided clinical expertise. HK designed the study, analyzed data and prepared the manuscript. All authors gave final approval to the manuscript.

\section{Acknowledgements}

This work was supported in part by grants from the National Heart, Lung and Blood Institute (HL0643 IO to H.K.), the National Human Genome Research Institute (HGOI577 to K.G.) and the National Center for Research Resources (RR03655).

\section{References}

I. Steinmetz EF, Buckley C, Thompson RW: Prospects for the medical management of abdominal aortic aneurysms. Vasc Endovascular Surg 2003, 37(3): $15 \mid-163$.

2. Hirose H, Tilson MD: Abdominal aortic aneurysm as an autoimmune disease. Ann N Y Acad Sci 200I, 947:4I6-4I8.

3. Brophy CM, Reilly JM, Smith G], Tilson MD: The role of inflammation in nonspecific abdominal aortic aneurysm disease. Ann Vasc Surg 1991, 5(3):229-233.

4. Koch AE, Haines GK, Rizzo RJ, Radosevich JA, Pope RM, Robinson PG, Pearce WH: Human abdominal aortic aneurysms. Immunophenotypic analysis suggesting an immune-mediated response. Am J Pathol 1990, 137(5): I 199-1213.

5. Price P, Witt C, Allcock R, Sayer D, Garlepp M, Kok CC, French M, Mallal S, Christiansen F: The genetic basis for the association of the 8.I ancestral haplotype (AI, B8, DR3) with multiple immunopathological diseases. Immunol Rev 1999, 167:257-274.

6. Simmonds MJ, Howson JM, Heward JM, Cordell HJ, Foxall H, CarrSmith J, Gibson SM, Walker N, Tomer Y, Franklyn JA, Todd JA, Gough SC: Regression mapping of association between the human leukocyte antigen region and Graves disease. Am J Hum Genet 2005, 76(I):157-163.

7. Marsh SGE, Parham P, Barber LD: THE HLA Facts Book. San Diego , ACADEMIC PRESS; 2000: I-398.

8. Warrens A, Lechler R: HLA in health and disease. San Diego, Academic Press; 1999.

9. Trowsdale J, Powis SH: The MHC: relationship between linkage and function. Curr Opin Genet Dev 1992, 2(3):492-497.

10. Altmann DM: HLA-DQ associations with autoimmune disease. Autoimmunity 1992, I 4(I):79-83.

II. Norrgard O, Cedergren B, Angquist KA, Beckman L: Blood groups and HLA antigens in patients with abdominal aortic aneurysms. Hum Hered 1984, 34(1):9-13.

12. Tilson MD, Ozsvath KJ, Hirose $H$, Xia S: A genetic basis for autoimmune manifestations in the abdominal aortic aneurysm resides in the MHC class II locus DR-beta-I. Ann N Y Acad Sci 1996, 800:208-2I5.

13. Rasmussen TE, Hallett JWJ, Metzger RL, Richardson DM, Harmsen WS, Goronzy JJ, Weyand CM: Genetic risk factors in inflammatory abdominal aortic aneurysms: polymorphic residue $\mathbf{7 0}$ in the HLA-DR B I gene as a key genetic element. J Vasc Surg 1997, 25(2):356-364.

14. Hirose H, Takagi M, Miyagawa N, Hashiyada H, Noguchi M, Tada S, Kugimiya T, Tilson MD: Genetic risk factor for abdominal aortic aneurysm: HLA-DR2(15), a Japanese study. J Vasc Surg 1998, 27(3):500-503.

15. Hirose H, Tilson MD: Negative genetic risk factor for abdominal aortic aneurysm: HLA-DQ3, a Japanese study. J Vasc Surg 1999, 30(5):959-960.

16. Rasmussen TE, Hallett JWJ, Schulte S, Harmsen WS, O'Fallon WM, Weyand CM: Genetic similarity in inflammatory and degenerative abdominal aortic aneurysms: a study of human leukocyte antigen class II disease risk genes. J Vasc Surg 200I, 34(I):84-89.

17. Rasmussen TE, Hallett JWJ, Tazelaar HD, Miller VM, Schulte S, O'FalIon WM, Weyand CM: Human leukocyte antigen class II immune response genes, female gender, and cigarette smoking as risk and modulating factors in abdominal aortic aneurysms. J Vasc Surg 2002, 35(5):988-993.

18. Sugimoto T, Sada M, Miyamoto T, Yao H: Genetic analysis on HLA loci in Japanese patients with abdominal aortic aneurysm. Eur J Vasc Endovasc Surg 2003, 26(2):2I5-2I8.

19. Monux G, Serrano FJ, Vigil P, De la Concha EG: Role of HLA-DR in the pathogenesis of abdominal aortic aneurysm. Eur J Vasc Endovasc Surg 2003, 26(2):2। I-2I4.

20. Johnston KW, Rutherford RB, Tilson MD, Shah DM, Hollier L, Stanley JC: Suggested standards for reporting on arterial aneurysms. Subcommittee on Reporting Standards for Arterial Aneurysms, Ad Hoc Committee on Reporting Standards, Society 
for Vascular Surgery and North American Chapter, International Society for Cardiovascular Surgery. J Vasc Surg 1991, 13(3):452-458.

21. Baird PA, Sadovnick AD, Yee IM, Cole CW, Cole L: Sibling risks of abdominal aortic aneurysm. Lancet 1995, 346(8975):60I-604.

22. Lawrence PF, Wallis C, Dobrin PB, Bhirangi K, Gugliuzza N, Galt S, Kraiss L: Peripheral aneurysms and arteriomegaly: is there a familial pattern? J Vasc Surg 1998, 28(4):599-605.

23. Lederle $F A$, Johnson GR, Wilson SE: Abdominal aortic aneurysm in women. J Vasc Surg 200I, 34(I): I22-I26.

24. Kuivaniemi $H$, Yoon S, Shibamura H, Skunca M, Vongpunsawad S, Tromp G: Primer-extension preamplified DNA is a reliable template for genotyping. Clin Chem 2002, 48(9): I60I-1604.

25. HUGO Gene Nomenclature Committee. [http://
[ www.gene.ucl.ac.uk/nomenclature]

26. Kimura A, Dong RP, Harada H, Sasazuki T: DNA typing of HLA class II genes in B-lymphoblastoid cell lines homozygous for HLA. Tissue Antigens 1992, 40(I):5-12.

27. Gregoire L, Lawrence WD, Kukuruga $D$, Eisenbrey $A B$, Lancaster WD: Association between HLA-DQB I alleles and risk for cervical cancer in African-American women. Int J Cancer 1994, 57(4):504-507.

28. Sham PC, Curtis D: Monte Carlo tests for associations between disease and alleles at highly polymorphic loci. Ann Hum Genet 1995, 59 ( Pt I):97-105.

29. Schaid DJ, Rowland CM, Tines DE, Jacobson RM, Poland GA: Score tests for association between traits and haplotypes when linkage phase is ambiguous. Am J Hum Genet 2002, 70(2):425-434.

30. Abecasis GR, Cookson WO: GOLD--graphical overview of linkage disequilibrium. Bioinformatics 2000, 16(2): I82-183.

31. Ogata T, Shibamura H, Tromp G, Sinha M, Goddard KA, Sakalihasan N, Limet R, MacKean GL, Arthur C, Sueda T, Kuivaniemi H: Genetic analysis of polymorphisms in biologically relevant candidate genes in patients with abdominal aortic aneurysms. J Vasc Surg 2005, 41: 1036-1042.

32. Ernst CB: Abdominal aortic aneurysm. N Engl J Med 1993, 328(16): I I67-II72.

33. St Leger AS, Spencely M, McCollum CN, Mossa M: Screening for abdominal aortic aneurysm: a computer assisted cost-utility analysis. Eur J Vasc Endovasc Surg 1996, II (2): 183-190.

34. Wilmink $A B$, Quick $C R$ : Epidemiology and potential for prevention of abdominal aortic aneurysm. $\mathrm{Br} J$ Surg 1998, 85(2): $155-162$.

35. Majumder PP, St Jean PL, Ferrell RE, Webster MW, Steed DL: On the inheritance of abdominal aortic aneurysm. Am J Hum Genet 1991, 48(I): 164-170.

36. Verloes A, Sakalihasan N, Koulischer L, Limet R: Aneurysms of the abdominal aorta: familial and genetic aspects in three hundred thirteen pedigrees. J Vasc Surg 1995, 2 I (4):646-655.

37. Kuivaniemi H, Shibamura $\mathrm{H}$, Arthur $\mathrm{C}$, Berguer R, Cole CW, Juvonen T, Kline RA, Limet R, Mackean G, Norrgard O, Pals G, Powell JT, Rainio P, Sakalihasan N, van Vlijmen-van Keulen C, Verloes A, Tromp G: Familial abdominal aortic aneurysms: collection of 233 multiplex families. J Vasc Surg 2003, 37(2):340-345.

38. Shibamura $\mathrm{H}$, Olson JM, van Vlijmen-Van Keulen $\mathrm{C}$, Buxbaum SG, Dudek DM, Tromp G, Ogata T, Skunca M, Sakalihasan N, Pals G, Limet R, MacKean GL, Defawe O, Verloes A, Arthur C, Lossing AG Burnett M, Sueda T, Kuivaniemi H: Genome scan for familial abdominal aortic aneurysm using sex and family history as covariates suggests genetic heterogeneity and identifies linkage to chromosome 19q I3. Circulation 2004, 109(17):2103-2108.

39. Campbell $C D$, Ogburn EL, Lunetta KL, Lyon HN, Freedman ML, Groop LC, Altshuler D, Ardlie KG, Hirschhorn JN: Demonstrating stratification in a European American population. Nat Genet 2005, 37(8):868-872.

40. Helgason A, Yngvadottir B, Hrafnkelsson B, Gulcher J, Stefansson K: An Icelandic example of the impact of population structure on association studies. Nat Genet 2005, 37(I):90-95.

4I. Redondo MJ, Fain PR, Eisenbarth GS: Genetics of type IA diabetes. Recent Prog Horm Res 200I, 56:69-89.

42. Marchini M, Antonioli R, Lleo A, Barili M, Caronni M, Origgi L, Vanoli M, Scorza R: HLA class II antigens associated with lupus nephritis in Italian SLE patients. Hum Immunol 2003, 64(4):462-468.
43. Beck S, Trowsdale J: The human major histocompatability complex: lessons from the DNA sequence. Annu Rev Genomics Hum Genet 2000, I:I I7-137.

44. Beauchamp GK, Yamazaki K: HLA and mate selection in humans: commentary. Am J Hum Genet 1997, 6 I (3):494-496.

\section{Pre-publication history}

The pre-publication history for this paper can be accessed here:

http://www.biomedcentral.com/1471-2350/7/67/prepub
Publish with Biomed Central and every scientist can read your work free of charge

"BioMed Central will be the most significant development for disseminating the results of biomedical research in our lifetime. "

Sir Paul Nurse, Cancer Research UK

Your research papers will be:

- available free of charge to the entire biomedical community

- peer reviewed and published immediately upon acceptance

- cited in PubMed and archived on PubMed Central

- yours - you keep the copyright

Submit your manuscript here:

http://www.biomedcentral.com/info/publishing_adv.asp
BiolMedcentral 